Document downloaded from:

http://hdl.handle.net/10251/62895

This paper must be cited as:

Calbo Sanjuán, G.; Cortés López, JC.; Jódar Sánchez, LA. (2011). Random Hermite differential equations: Mean square power series solutions and statistical properties. Applied Mathematics and Computation. 218(7):3654-3666. doi:10.1016/j.amc.2011.09.008.

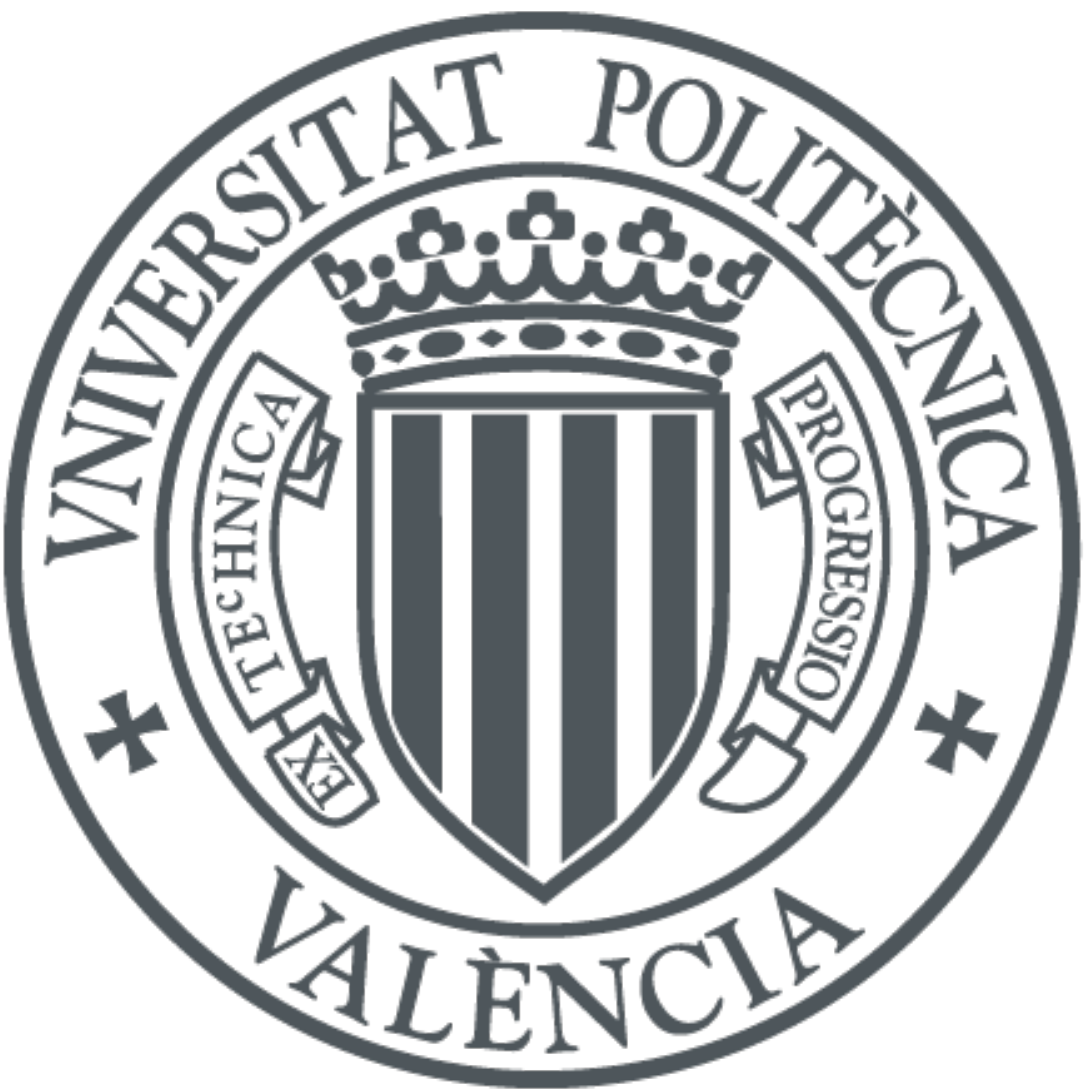

The final publication is available at

http://dx.doi.org/10.1016/j.amc.2011.09.008

Copyright Elsevier

Additional Information 


\title{
Random Hermite differential equations: Mean square power series solutions and statistical properties
}

\author{
G. Calbo, J.-C. Cortés*, L. Jódar \\ Instituto Universitario de Matemática Multidisciplinar, \\ Universitat Politècnica de València, \\ Camino de Vera s/n, Building 8G, $2^{\text {nd }}$ floor, 46022 Valencia, SPAIN
}

\begin{abstract}
This paper deals with the construction of random power series solution of second order linear differential equations of Hermite containing uncertainty through its coefficients and initial conditions. Under appropriate hypotheses on the data, we establish that the constructed random power series solution is mean square convergent. We provide conditions in order to obtain random polynomial solutions and, as a consequence, random Hermite polynomial are introduced. Also, the main statistical functions of the approximate stochastic process solution generated by truncation of the exact power series solution are given. Finally, we apply the proposed technique to several illustrative examples comparing the numerical results with respect to those provided by other available approaches including Monte Carlo simulation.
\end{abstract}

Key words: random differential equation, random power series solution, mean square calculus, random Hermite polynomial

\section{Introduction}

Differential equations are powerful tools to represent reality up to certain point. Real problems use to involve a combination of complexity, uncertainty

\footnotetext{
* Corresponding author.

Email addresses: gcalbo@imm.upv.es (G. Calbo), jccortes@imm.upv.es phone: 0034-963877000 (ext.88289) (J.-C. Cortés), ljodar@imm.upv.es (L. Jódar).
} 
and/or ignorance, sometimes due to natural phenomena, and also due to human behaviour. The quantification of uncertainty requires a model specifying the mechanism by which randomness is generated.

Random differential equations have been used in the last few decades to deal with errors and uncertainty. For example, see [1] for the general randomness case and $[2,3]$ for the case of white noise uncertainty. Theoretical approaches of random differential equations probably started with Strand in $[4,1]$. Thinking of applications using explicit analytic solutions or numerical methods, a few results may be found in $[5-11]$.

Hermite differential equation has a great interest in the development of applied mathematics, not only because it appears in a natural way when solving partial differential equations of the mathematical physics by using the separation of variables technique, but also because its prevalent role in other areas of mathematics such as functional analysis or orthogonal polynomials theory. The aim of this paper is twofold. Firstly, constructing mean square series solutions of the random Hermite differential equation

$$
\ddot{X}(t)-2 t \dot{X}(t)+A X(t)=0, \quad-\infty<t<+\infty,
$$

where $A$ is a random variable satisfying certain conditions to be specified later. Secondly, we show how and under which situations the random Hermite polynomials appear and are solutions of appropriate random Hermite differential equations. An important difficulty to be overcome is the lack of sub-multiplicativity of the mean square norm (and as a consequence of the mean fourth norm) as well as the necessity of bounding products of random variables. Particularly interesting is the case where $A$ is a discrete random variable taking a finite number of even integer values. In such case, it appears mean square finite series solutions of problem (1), referred as to random Hermite polynomials.

The paper is organized as follows. Section 2 deals with some preliminaries about the mean square calculus that will be required throughout the paper. The concepts of fundamental set of solution process to equation (1) as well as random polynomial are also introduced in Section 2. Section 3 deals with the proof of an important inequality related to the norm of the product of random variables. This result manages satisfactorily the lack of submultiplicativity of the mean square and mean fourth norms. Section 4 deals with the construction of mean square convergent power series solution to (1) in the case where $A$ is a random variable satisfying certain conditions related to the exponential growth of its absolute moments with respect to the origin. The case where $A$ is a discrete random variable taking a finite number of even values leads to the concept of random Hermite polynomials whose definition and obtention is shown in Section 5. Statistical functions of the truncated random power series solutions, and particularly of random Hermite polynomials, are studied 
in Section 6. Finally, in Section 7 some illustrative examples are presented showing the discrepancies with respect to the so-called dishonest and Monte Carlo methods.

\section{Preliminaries}

For the sake of clarity in the presentation, we begin this section by introducing some concepts, notations and results that may be found in [5, chap.4], [12, part IV], [13, chap.1-3]. Let $(\Omega, \mathcal{F}, P)$ be a probability space. In this paper we will work in the set $L_{2}$ which elements are second order real random variables (2-r.v.'s), i.e., $X: \Omega \rightarrow \mathbb{R}$ such that $\mathrm{E}\left[X^{2}\right]<+\infty$, where $\mathrm{E}[\cdot]$ denotes the expectation operator. One can demonstrate that $L_{2}$ endowed with the socalled 2-norm

has a Banach space structure.

$$
\|X\|_{2}=\left(\mathrm{E}\left[X^{2}\right]\right)^{1 / 2}
$$

As it is usual, given a r.v. $X, \mathrm{E}\left[X^{k}\right], k=0,1,2, \ldots$ and $\mathrm{E}\left[|X|^{s}\right], s>0$ will denote the $k$-th moment and $s$-th absolute moment (both with respect to the origin), respectively. Note that, $\mathrm{E}\left[X^{0}\right]=\mathrm{E}\left[|X|^{0}\right]=1$. It is easy to prove that

$$
\text { if } \mathrm{E}\left[|X|^{s}\right]<+\infty \Rightarrow \begin{cases}\exists \mathrm{E}\left[|X|^{r}\right]<+\infty & \forall r: 0 \leq r \leq s, \\ \exists \mathrm{E}\left[X^{m}\right]<+\infty & \forall m: m=0,1, \ldots, s .\end{cases}
$$

Next result, so-called $c_{s}$-inequality, is useful for bounding the absolute moments of a binomial expression in terms of the absolute moments of both summands and moreover it establishes that if $s$-th absolute moments of $X$ and $Y$ are finite then $s$-th absolute moment of $X+Y$ does

$$
\mathrm{E}\left[|X+Y|^{s}\right] \leq c_{s}\left(\mathrm{E}\left[|X|^{s}\right]+\mathrm{E}\left[|Y|^{s}\right]\right), \quad c_{s}= \begin{cases}1 & \text { if } s \leq 1, \\ 2^{s-1} & \text { if } s \geq 1 .\end{cases}
$$

We say that $\{X(t): t \in \mathcal{T}\}$ is a second order stochastic process (2-s.p.), if the r.v. $X(t) \in L_{2}$ for each $t \in \mathcal{T}$, being $\mathcal{T}$ the so-called space of times. Throughout this paper we will assume that $\mathcal{T}$ is always a real interval. The expectation function of $X(t)$ provides a statistical measure of its mean statistical behavior and it will denoted by $\mathrm{E}[X(t)]$ or $\mu_{X}(t)$, while its covariance function will be denoted by $\operatorname{Cov}[X(t), X(s)]$ and it is defined as follows

$$
\begin{aligned}
\operatorname{Cov}[X(t), X(s)] & =\mathrm{E}\left[\left(X(t)-\mu_{X}(t)\right)\left(X(s)-\mu_{X}(s)\right)\right] \\
& =\mathrm{E}[X(t) X(s)]-\mu_{X}(t) \mu_{X}(s), \quad t, s \in \mathcal{T} .
\end{aligned}
$$


When $s=t$, one obtains the variance function

$$
\operatorname{Var}[X(t)]=\operatorname{Cov}[X(t), X(t)]=\mathrm{E}\left[(X(t))^{2}\right]-\left(\mu_{X}(t)\right)^{2} .
$$

The term $\Gamma_{X}(t, s)=\mathrm{E}[X(t) X(s)]$ appearing into (5) is called the correlation function and it plays an important role in the m.s. calculus because many important stochastic results can be characterized through this two-variables deterministic function (see, [5, chap.4]).

We say that a sequence of 2-r.v.'s $\left\{X_{n}: n \geq 0\right\}$ is mean square (m.s.) convergent to $X \in L_{2}$ if

$$
\lim _{n \rightarrow \infty}\left\|X_{n}-X\right\|_{2}=\lim _{n \rightarrow \infty}\left(\mathrm{E}\left[\left(X_{n}-X\right)^{2}\right]\right)^{1 / 2}=0 .
$$

Later we will present a method to provide approximations of the s.p. solution $X(t)$ to the random differential equation (1). The following properties will play a fundamental role when we are interested in computing the mean and variance of such approximations as well as assuring they are close to the correspondent exact values.

Lemma 1 (see, [5, p.88]) Let $\left\{X_{n}: n \geq 0\right\}$ be a sequence of 2-r.v.'s m.s. convergent to $X$, then

$$
\mathrm{E}\left[X_{n}\right] \underset{n \rightarrow \infty}{\longrightarrow} \mathrm{E}[X], \quad \operatorname{Var}\left[X_{n}\right] \underset{n \rightarrow \infty}{\longrightarrow} \operatorname{Var}[X]
$$

We say that a 2-s.p. $\{X(t): t \in \mathcal{T}\}$ is m.s. continuous in $\mathcal{T}$ if

$$
\lim _{\tau \rightarrow 0}\|X(t+\tau)-X(t)\|_{2}=0,
$$

for each $t \in \mathcal{T}$, such that $t+\tau \in \mathcal{T}$.

Example 2 Let $\left\{X_{n}: n \geq 1\right\}$ be a sequence of r.v.'s in $L_{2}$ and $t \in \mathcal{T}$ with $\mathcal{T}$ a real interval, then for each positive integer $n_{0}$, the 2-s.p. $\left\{n_{0} X_{n_{0}} t^{n_{0}-1}: t \in \mathcal{T}\right\}$ is m.s. continuous for all $t \in \mathcal{T}$. In fact,

$$
\left\|n_{0} X_{n_{0}}(t+\tau)^{n_{0}-1}-n_{0} X_{n_{0}} t^{n_{0}-1}\right\|_{2}=n_{0}\left|(t+\tau)^{n_{0}-1}-t^{n_{0}-1}\right|\left\|X_{n_{0}}\right\|_{2} \underset{\tau \rightarrow 0}{\longrightarrow} 0,
$$

because $\left\|X_{n_{0}}\right\|_{2}<+\infty$ as $X_{n_{0}} \in L_{2}$ for each $n_{0}$ and, the continuity of the deterministic function $f(t)=t^{n_{0}-1}$ with respect to $t$.

A 2-s.p. $\{X(t): t \in \mathcal{T}\}$ is said to be m.s. differentiable at $t \in \mathcal{T}$ and $\dot{X}(t)$ denotes its $\mathrm{m}$.s. derivative if

$$
\lim _{\tau \rightarrow 0}\left\|\frac{X(t+\tau)-X(t)}{\tau}-\dot{X}(t)\right\|_{2}=0,
$$

for all $t \in \mathcal{T}$, such that $t+\tau \in \mathcal{T}$. 
Example 3 With the notation of Example 2, the process $\left\{X_{n_{0}} t^{n_{0}}: t \in \mathcal{T}\right\}$ is m.s. differentiable in $\mathcal{T}$ and its m.s. derivative is $\left\{n_{0} X_{n_{0}} t^{n_{0}-1}: t \in \mathcal{T}\right\}$. Note that,

$$
\left\|\frac{X_{n_{0}}(t+\tau)^{n_{0}}-X_{n_{0}} t^{n_{0}}}{\tau}-n_{0} X_{n_{0}} t^{n_{0}-1}\right\|_{2}=\left|\frac{(t+\tau)^{n_{0}}-t^{n_{0}}}{\tau}-n_{0} t^{n_{0}-1}\right|\left\|X_{n_{0}}\right\|_{2} \underset{\tau \rightarrow 0}{\longrightarrow} 0 .
$$

Later, due to the random differential equation (1) involves m.s. derivatives up to second order, the following result will required. For $\{X(t): t \in \mathcal{T}\}$ a 2-s.p. twice m.s. differentiable on $\mathcal{T}$, one can demonstrate that the averages of its two first m.s. derivatives exist and they are given by

$$
\mathrm{E}[\dot{X}(t)]=\frac{d}{d t}(\mathrm{E}[X(t)]), \quad \mathrm{E}[\ddot{X}(t)]=\frac{d^{2}}{d t^{2}}(\mathrm{E}[X(t)]), \quad \forall t \in \mathcal{T}
$$

where $\frac{d}{d t}$ and $\frac{d^{2}}{d t^{2}}$ denote first and second deterministic derivatives, respectively. This result can be extended to the $n$-th m.s. derivative whenever $\{X(t): t \in \mathcal{T}\}$ is $n$-th times m.s. differentiable on $\mathcal{T}$ (see [5, p.97]).

If $X$ and $Y$ are 2-r.v.'s, Schwarz inequality establishes that

$$
\mathrm{E}[|X Y|] \leq\left(\mathrm{E}\left[X^{2}\right]\right)^{1 / 2}\left(\mathrm{E}\left[Y^{2}\right]\right)^{1 / 2}
$$

Later we will require the following basic property

$$
A X_{n} \underset{n \rightarrow \infty}{\stackrel{\text { m.s. }}{\longrightarrow}} A X
$$

which holds true if $A \in L_{2},\left\{X_{n}: n \geq 0\right\}$ is a sequence of 2-r.v.'s such that $X_{n} \underset{n \rightarrow \infty}{\stackrel{\text { m.s. }}{\longrightarrow}} X$ and $A, X_{n}$ are independent r.v.'s for each $n$. However, independence hypothesis cannot be assumed in many practical cases like those that we will consider below. This motivates the introduction of r.v.'s $X$ such that $\mathrm{E}\left[X^{4}\right]<\infty$ which will be denoted by 4-r.v.'s. Note that a 4-r.v. is a 2-r.v. The set $L_{4}$ of all the 4-r.v.'s endowed with the norm

$$
\|X\|_{4}=\sqrt[4]{\mathrm{E}\left[X^{4}\right]}
$$

is a Banach space (see [2, p.9]). A stochastic process $\{X(t): t \in T\}$, where $\mathrm{E}\left[(X(t))^{4}\right]<\infty$ for all $t \in T$, will be called a 4-s.p.

Definition 4 A sequence of 4-r.v.'s $\left\{X_{n}: n \geq 0\right\}$ is said to be mean fourth (m.f.) convergent to a 4-r.v. $X$ if

$$
\lim _{n \rightarrow \infty}\left\|X_{n}-X\right\|_{4}=0 .
$$

This type of convergence will be represented by $X_{n} \underset{n \rightarrow \infty}{\stackrel{m . f .}{\longrightarrow}} X$. 
The following lemma establishes the link between the two types of convergence introduced previously.

Lemma 5 Let $\left\{X_{n}: n \geq 0\right\}$ be a sequence of 4-r.v.'s and suppose that $X_{n} \underset{n \rightarrow \infty}{\stackrel{m . f .}{\longrightarrow}} X$. Then $X_{n} \underset{n \rightarrow \infty}{\stackrel{m . s .}{\longrightarrow}} X$.

Proof. Using the Schwarz inequality (9), one gets

$$
\left(\left\|X_{n}-X\right\|_{2}\right)^{2}=\mathrm{E}\left[1 \times\left(X_{n}-X\right)^{2}\right] \leq 1 \times\left(\mathrm{E}\left[\left(X_{n}-X\right)^{4}\right]\right)^{1 / 2}=\left(\left\|X_{n}-X\right\|_{4}\right)^{2} .
$$

Since $\left\|X_{n}-X\right\|_{4} \underset{n \rightarrow+\infty}{\longrightarrow} 0$ (because $X_{n}$ is m.f. convergent to $X$ ), it immediately follows that $\left\|X_{n}-X\right\|_{2} \underset{n \rightarrow \infty}{\longrightarrow} 0$ and therefore $X_{n} \underset{n \rightarrow \infty}{\stackrel{\text { m.s. }}{\longrightarrow}} X$.

Now, we can give sufficient conditions in order to property (10) holds true without assuming hypotheses based on independence.

Lemma 6 Let $A$ be a 4-r.v. and $\left\{X_{n}: n \geq 0\right\}$ a sequence of 4-r.v.'s such that $X_{n} \underset{n \rightarrow \infty}{\stackrel{m . f .}{\longrightarrow}} X$. Then $A X_{n} \underset{n \rightarrow \infty}{\stackrel{m . s .}{\longrightarrow}} A X$.

Proof. By the definition of the norm $\|\cdot\|_{2}$, we have

$$
\left(\left\|A\left(X_{n}-X\right)\right\|_{2}\right)^{2}=\mathrm{E}\left[A^{2}\left(X_{n}-X\right)^{2}\right] .
$$

On the other hand, the hypothesis $X_{n} \underset{n \rightarrow \infty}{\stackrel{\text { m.f. }}{\longrightarrow}} X$ implies by definition $\left(X_{n}-\right.$ $X)^{2} \underset{n \rightarrow \infty}{\stackrel{\text { m.s. }}{\longrightarrow}} 0$ and, as clearly $A^{2} \underset{n \rightarrow \infty}{\stackrel{\text { m.s. }}{\longrightarrow}} A^{2}$, then from (12) and the Lemma 1 one obtains $\left\|A\left(X_{n}-X\right)\right\|_{2} \underset{n \rightarrow \infty}{\longrightarrow} 0$ and hence $A X_{n} \underset{n \rightarrow \infty}{\stackrel{\text { m.s. }}{\longrightarrow}} A X$.

Next we extent from the deterministic framework to the random one the concept of fundamental set of solutions.

Definition 7 Let $A_{1}$ and $A_{2}$ be r.v.'s, and let $X_{1}(t)$ and $X_{2}(t)$ a pair of solutions of the second-order random differential equation

$$
\ddot{X}(t)+A_{1} \dot{X}(t)+A_{2} X(t)=0, \quad-\infty<t<\infty .
$$

We say that $\left\{X_{1}(t), X_{2}(t)\right\}$ is a fundamental set of solution processes of (13) in $-\infty<t<+\infty$, if any solution $X(t)$ of (13) admits a unique representation of the form

$$
X(t)=C_{1} X_{1}(t)+C_{2} X_{2}(t), \quad t \in(-\infty, \infty),
$$

where $C_{1}$ and $C_{2}$ are r.v.'s uniquely determined by $X(t)$.

Definition 8 Let $\mathcal{S}=\left\{X_{1}(t), X_{2}(t)\right\}$ be a pair of solutions of (13), the s.p. given by

$$
W_{\mathcal{S}}(t)=X_{1}(t) \dot{X}_{2}(t)-X_{2}(t) \dot{X}_{1}(t),
$$


is called the wronskian determinant process of $\mathcal{S}$.

The following result provides sufficient conditions for a pair of solutions of (13) defines a fundamental set. We omit the proof because it follows in broad outline the same steps as in the deterministic case.

Proposition 9 If $\mathcal{S}=\left\{X_{1}(t), X_{2}(t)\right\}$ is a pair of solution processes of the random differential equation (13) in $-\infty<t<\infty$ and there exists $t_{0} \in$ $(-\infty, \infty)$ such that $W_{\mathcal{S}}\left(t_{0}\right) \neq 0$, then $\mathcal{S}=\left\{X_{1}(t), X_{2}(t)\right\}$ is a fundamental set of solution processes of (13).

As we have outlined in the first section, we are interested in determining solutions of differential equation (1) by means of random power series which under certain conditions become random polynomials. Now we introduce these concepts.

Definition 10 Given a collection of r.v.'s $\left\{X_{k}: k \geq 0\right\}$ and $t \in \mathcal{T}, \sum_{k \geq 0} X_{k} t^{k}$ is called a random power series. If $P\left[X_{k}=0\right]=1$ for all $k>m$, that is, $P\left[\left\{\omega \in \Omega: X_{k}(\omega)=0, \forall k>m\right\}\right]=1$, then $\sum_{k=0}^{m} X_{k} t^{k}$ is said to be a random polynomial in $t$ of degree $m$.

We close this section by recalling the following differentiation theorem for m.s. random convergent series that will be required later.

Theorem 11 (see, [18]) Let us assume that for each integer $k \geq 0$, the s.p. $\left\{U_{k}(t): t \in \mathcal{T}\right\}$ is m.s. differentiable for each $t \in \mathcal{T},\left\{\dot{U}_{k}(t): t \in \mathcal{T}\right\}$ is m.s. continuous, $U(t)=\sum_{k \geq 0} U_{k}(t)$ is m.s. convergent and the series $\sum_{k \geq 0} \dot{U}_{k}(t)$ is m.s. uniformly convergent. Then the s.p. $\{U(t): t \in \mathcal{T}\}$ is m.s. differentiable and

$$
\dot{U}(t)=\sum_{k \geq 0} \dot{U}_{k}(t)
$$

\section{A crucial inequality}

An important fact is that the 2 -norm $\|\cdot\|_{2}$ given by (2) does not provide a Banach algebra structure to $L_{2}$, i.e., it is not submultiplicative because the property $\|X Y\|_{2} \leq\|X\|_{2}\|Y\|_{2}$ does not hold. In fact, let $Z$ be a non-constant positive 2-r.v. and let us take $X=Y=Z^{1 / 2}$, then

$$
\left(\|X Y\|_{2}\right)^{2}-\left(\|X\|_{2}\right)^{2}\left(\|Y\|_{2}\right)^{2}=\mathrm{E}\left[Z^{2}\right]-(\mathrm{E}[Z])^{2}=\operatorname{Var}[Z]>0 .
$$

Therefore $\|X Y\|_{2}>\|X\|_{2}\|Y\|_{2}$. As a consequence, 2-norm $\|\cdot\|_{2}$ is not submultiplicative. This situation difficult our next target. Indeed, in the following section we shall obtain the solution of (1) by a random power series in which 
the general term is the product of r.v.'s. In order to establish the absolute m.f. convergence of this series (thus its m.s. convergence), we will require to bound the 4-norm of a product of r.v.'s in terms of the 4-norm of each factor. Next we address our work to provide some results to overcome this difficulty.

Given a r.v. $X$ such that $\mathrm{E}\left[|X|^{n}\right]<+\infty$ then

$$
\left(\mathrm{E}\left[|X|^{m}\right]\right)^{1 / m} \leq\left(\mathrm{E}\left[|X|^{n}\right]\right)^{1 / n}, \quad 0 \leq m \leq n .
$$

Note that in this case, $\mathrm{E}\left[|X|^{m}\right]<\infty$ holds. This result is called the Liapounov's inequality (see, [12, p.157]).

Next proposition contains the main result in this section and it constitutes a generalization of (9).

Proposition 12 Let $\left\{X_{i}\right\}_{i=1}^{n}, n \geq 2$ be r.v.'s such that $\mathrm{E}\left[\left(X_{i}\right)^{2^{n}}\right]<\infty$, $i=1,2, \ldots, n$, then

$$
\mathrm{E}\left[\left|\prod_{i=1}^{n} X_{i}\right|\right] \leq\left(\prod_{i=1}^{n} \mathrm{E}\left[\left(X_{i}\right)^{2^{n-1}}\right]\right)^{1 / 2^{n-1}}, \quad n \geq 2 .
$$

Proof. It follows by induction on the number $n$ of the involved r.v.'s. For $n=2,(16)$ is exactly the Schwarz's inequality given by (9). Note that since by hypothesis $\mathrm{E}\left[\left(X_{i}\right)^{4}\right]<\infty$ for $i=1,2$, then by (3) one gets $\mathrm{E}\left[\left(X_{i}\right)^{2}\right]<\infty$, $i=1,2$. For $n=3$, by applying twice (9) one obtains

$$
\begin{aligned}
\mathrm{E}\left[\left|X_{1} X_{2} X_{3}\right|\right] & \leq\left(\mathrm{E}\left[\left(X_{1} X_{2}\right)^{2}\right]\right)^{1 / 2}\left(\mathrm{E}\left[\left(X_{3}\right)^{2}\right]\right)^{1 / 2} \\
& =\left(\mathrm{E}\left[\left(X_{1}\right)^{2}\left(X_{2}\right)^{2}\right]\right)^{1 / 2}\left(\mathrm{E}\left[\left(X_{3}\right)^{2}\right]\right)^{1 / 2} \\
& \leq\left(\mathrm{E}\left[\left(X_{1}\right)^{4}\right]\right)^{1 / 4}\left(\mathrm{E}\left[\left(X_{2}\right)^{4}\right]\right)^{1 / 4}\left(\mathrm{E}\left[\left(X_{3}\right)^{2}\right]\right)^{1 / 2} \\
& \leq\left(\mathrm{E}\left[\left(X_{1}\right)^{4}\right]\right)^{1 / 4}\left(\mathrm{E}\left[\left(X_{2}\right)^{4}\right]\right)^{1 / 4}\left(\mathrm{E}\left[\left(X_{3}\right)^{4}\right]\right)^{1 / 4},
\end{aligned}
$$

where in the last inequality we have applied (15) for $X=X_{3}$ and $m=2 \leq$ $4=n$. Now, we assume by induction hypothesis that (16) holds and we shall establish that

$$
\mathrm{E}\left[\left|\prod_{i=1}^{n+1} X_{i}\right|\right] \leq\left(\prod_{i=1}^{n+1} \mathrm{E}\left[\left(X_{i}\right)^{2^{n}}\right]\right)^{1 / 2^{n}} .
$$

Indeed, by the induction hypothesis one gets

$$
\begin{aligned}
\mathrm{E}\left[\left|\prod_{i=1}^{n+1} X_{i}\right|\right] & =\mathrm{E}\left[\left|\left(X_{1}\right)\left(X_{2}\right) \cdots\left(X_{n-1}\right)\left(X_{n} X_{n+1}\right)\right|\right] \\
& \leq\left(\mathrm{E}\left[\left(X_{1}\right)^{2^{n-1}}\right]\right)^{1 / 2^{n-1}}\left(\mathrm{E}\left[\left(X_{2}\right)^{2^{n-1}}\right]\right)^{1 / 2^{n-1}} \cdots\left(\mathrm{E}\left[\left(X_{n-1}\right)^{2^{n-1}}\right]\right)^{1 / 2^{n-1}} \\
& \times\left(\mathrm{E}\left[\left(X_{n}\right)^{2^{n-1}}\left(X_{n+1}\right)^{2^{n-1}}\right]\right)^{1 / 2^{n-1}},
\end{aligned}
$$


next we apply the Liapounov's inequality (15) to the $n-1$ first factors and, the Schwarz's inequality (9) to the last factor of the right-hand side, this yields

$$
\begin{aligned}
\mathrm{E}\left[\left|\prod_{i=1}^{n+1} X_{i}\right|\right] & \leq\left(\mathrm{E}\left[\left(X_{1}\right)^{2^{n}}\right]\right)^{1 / 2^{n}}\left(\mathrm{E}\left[\left(X_{2}\right)^{2^{n}}\right]\right)^{1 / 2^{n}} \cdots\left(\mathrm{E}\left[\left(X_{n-1}\right)^{2^{n}}\right]\right)^{1 / 2^{n}} \\
& \times\left(\mathrm{E}\left[\left(X_{n}\right)^{2^{n}}\right]\right)^{1 / 2^{n}}\left(\mathrm{E}\left[\left(X_{n+1}\right)^{2^{n}}\right]\right)^{1 / 2^{n}} \\
& =\left(\prod_{i=1}^{n+1} \mathrm{E}\left[\left(X_{i}\right)^{2^{n}}\right]\right)^{1 / 2^{n^{n}}}
\end{aligned}
$$

Thus the result is established.

Now we rewrite the inequality (16) in terms of the 4-norm given by (11). For this, we will assume that $\mathrm{E}\left[\left(Y_{i}\right)^{2^{n+1}}\right]<+\infty, i=1,2, \ldots, n$ and we apply Proposition 12 to the r.v.'s $X_{i}=\left(Y_{i}\right)^{4}, i=1,2, \ldots, n$, thus one obtains

$$
0<\mathrm{E}\left[\left(\prod_{i=1}^{n} Y_{i}\right)^{4}\right] \leq\left(\prod_{i=1}^{n} \mathrm{E}\left[\left(\left(Y_{i}\right)^{4}\right)^{2^{n-1}}\right]\right)^{1 / 2^{n-1}}=\prod_{i=1}^{n}\left(\mathrm{E}\left[\left(\left(Y_{i}\right)^{2^{n-1}}\right)^{4}\right]\right)^{1 / 2^{n-1}}
$$

taking fourth roots in this expression it follows

$$
\left(\mathrm{E}\left[\left(\prod_{i=1}^{n} Y_{i}\right)^{4}\right]\right)^{1 / 4} \leq\left(\prod_{i=1}^{n} \mathrm{E}\left[\left(\left(Y_{i}\right)^{2^{n-1}}\right)^{4}\right]\right)^{1 / 2^{n+1}}=\prod_{i=1}^{n}\left(\left(\mathrm{E}\left[\left(\left(Y_{i}\right)^{2^{n-1}}\right)^{4}\right]\right)^{1 / 4}\right)^{1 / 2^{n-1}}
$$

i.e., considering the 4-norm definition one gets

$$
\left\|\prod_{i=1}^{n} Y_{i}\right\|_{4} \leq \prod_{i=1}^{n}\left(\left\|\left(Y_{i}\right)^{2^{n-1}}\right\|_{4}\right)^{1 / 2^{n-1}}, \quad \mathrm{E}\left[\left(Y_{i}\right)^{2^{n+1}}\right]<+\infty, i=1, \ldots, n .
$$

\section{Solving the random Hermite differential equations}

This section is addressed to obtain the solution of the random differential equation (1) by means of a random power series as well as establishing its m.s. convergence. As we will see later in this section, inequality (17) will play a crucial role in order to prove this type of convergence. In the following we will assume that the absolute moments with respect to the origin of r.v. $A$ appearing into (1) increase at the most exponentially, i.e, there exist a nonnegative integer $n_{0}$ and positive constants $H$ and $M$ such that

$$
\mathrm{E}\left[|A|^{n}\right] \leq H M^{n}<+\infty, \quad \forall n \geq n_{0}
$$


Let us seek a formal solution process of problem (1) of the form

$$
X(t)=\sum_{n \geq 0} X_{n} t^{n}
$$

where coefficients $X_{n}$ are 2-r.v.'s. Assuming that $X(t)$ is termwise m.s. differentiable and applying Example 3, it follows that

$$
\begin{gathered}
\dot{X}(t)=\sum_{n \geq 1} n X_{n} t^{n-1}, \quad t \dot{X}(t)=\sum_{n \geq 1} n X_{n} t^{n}, \\
\ddot{X}(t)=\sum_{n \geq 2} n(n-1) X_{n} t^{n-2}=2 X_{2}+\sum_{n \geq 1}(n+2)(n+1) X_{n+2} t^{n} .
\end{gathered}
$$

By imposing that (19)-(21) satisfy (1), one gets

$$
A X_{0}+2 X_{2}+\sum_{n \geq 1}\left((n+2)(n+1) X_{n+2}+(A-2 n) X_{n}\right) t^{n}=0 .
$$

Therefore a candidate m.s. solution process of problem (1) can be obtained by imposing

$$
\left.\begin{array}{cc}
A X_{0}+2 X_{2} & =0 \\
(n+2)(n+1) X_{n+2}+(A-2 n) X_{n} & =0, n \geq 1,
\end{array}\right\}
$$

i.e.,

$$
X_{n+2}=\frac{(2 n-A)}{(n+2)(n+1)} X_{n}, \quad n \geq 0 .
$$

By a recursive reasoning, these coefficients $X_{n}$ can be computed as follows

$$
\begin{gathered}
X_{2 k+2}=\frac{(-A)(4-A)(8-A) \cdots(4 k-A)}{(2 k+2) !} X_{0}, \quad k \geq 0, \\
X_{2 k+3}=\frac{(2-A)(6-A)(10-A) \cdots(4 k+2-A)}{(2 k+3) !} X_{1}, \quad k \geq 0 .
\end{gathered}
$$

Note that, all above equalities must be understanding with probability 1 . Therefore taking into account relationships (24)-(25), the s.p. (19) can be expressed in terms of the data

$$
X(t)=X_{0} X_{1}(t)+X_{1} X_{2}(t)
$$

where

$$
\begin{aligned}
& X_{1}(t)=\left(1+\sum_{k \geq 0} \frac{t^{2 k+2}}{(2 k+2) !} \prod_{j=0}^{k}(4 j-A)\right), \\
& X_{2}(t)=\left(t+\sum_{k \geq 0} \frac{t^{2 k+3}}{(2 k+3) !} \prod_{j=0}^{k}(4 j+2-A)\right) .
\end{aligned}
$$

Note that in (22) we have formally commuted r.v. $A$ and a random infinite sum, then property (10) has been applied implicitly. In order to legitimate 
this commutation, hypotheses of Lemma 6 must be checked. Therefore, we address our work to establish the m.f. convergence of the two random series given by $(27)$, and since $\left(L_{4},\|\cdot\|_{4}\right)$ is a Banach space, that is equivalent to prove that both series are absolutely convergent in the 4-norm. Thus, for each $t \in(-\infty,+\infty)$, we consider the numerical series associated to the first series in $(27)$ given by

$$
\sum_{k \geq 0} \frac{|t|^{2 k+2}}{(2 k+2) !}\left\|\prod_{j=0}^{k}(4 j-A)\right\|_{4},
$$

and note that by hypothesis $\mathrm{E}\left[|A|^{n}\right]<+\infty, \forall n \geq 0$, then applying (17) one gets

$$
\left\|\prod_{j=0}^{k}(4 j-A)\right\|_{4} \leq \prod_{j=0}^{k}\left(\left\|(4 j-A)^{2^{k}}\right\|_{4}\right)^{\frac{1}{2^{k}}} .
$$

Now we can bound each factor of the above right-hand side by considering (4). Indeed, let $k$ be a fixed nonnegative integer and, for each $j=0,1, \ldots, k$ let us consider the factor

$$
\begin{aligned}
\left(\left\|(4 j-A)^{2^{k}}\right\|_{4}\right)^{\frac{1}{2^{k}}} & =\left(\mathrm{E}\left[|4 j-A|^{2^{k+2}}\right]\right)^{\frac{1}{2^{k+2}}} \\
& \leq\left(2^{2^{k+2}-1}\left((4 j)^{2^{k+2}}+\mathrm{E}\left[|A|^{2^{k+2}}\right]\right)\right)^{\frac{1}{2^{k+2}}} \\
& \leq\left(2^{2^{k+2}-1}\left((4 k)^{2^{k+2}}+\mathrm{E}\left[|A|^{2^{k+2}}\right]\right)\right)^{\frac{1}{2^{k+2}}} .
\end{aligned}
$$

Under hypothesis (18), we can assure the existence of an integer, say, $k_{0}$ such that: $(4 k)^{2^{k+2}} \geq H M^{2^{k+2}}$ for each $k \geq k_{0}$, so from (30) one gets

$$
\begin{aligned}
\left(\left\|(4 j-A)^{2^{k}}\right\|_{4}\right)^{\frac{1}{2^{k}}} & \leq\left(2^{2^{k+2}-1}\left((4 k)^{2^{k+2}}+\mathrm{E}\left[|A|^{2^{k+2}}\right]\right)\right)^{\frac{1}{2^{k+2}}} \\
& \leq\left(2^{2^{k+2}}\left((4 k)^{2^{k+2}}\right)\right)^{\frac{1}{2^{k+2}}}=8 k, \quad \forall k \geq k_{0} .
\end{aligned}
$$

As we are only interested in convergence, we can assume without loss of generality that $k_{0}=0$. Then series (28) can be majorized by series

$$
\sum_{k \geq 0} \frac{|t|^{2 k+2}}{(2 k+2) !}(8 k)^{k+1},
$$

which is convergent for all $t$ as it can be directly checked by D'Alembert test. Therefore, we have proven that numerical series given by (28) is convergent, thus the first random series of (27) is m.f. convergent, so also m.s. convergent. Following an analogous procedure, it is easy to establish the m.s. convergence of the second series in (27) for all $t$. Note that the above reasoning shows that both solution series $X_{1}(t)$ and $X_{2}(t)$ given by (27) are m.s. uniformly convergent, therefore taking into account Examples 2 and 3 and Proposition 11 , the formal differentiation considered in (20)-(21) is justified. On the other hand, taking $t_{0}=0$ and considering that $X_{1}(0)=1, \dot{X}_{1}(0)=0, X_{2}(0)=0$ and $\dot{X}_{2}(0)=1$, one gets that $W_{\mathcal{S}}(0)=1 \neq 0$, then by Proposition 9 and (14), 
the solution of random differential equation (1) with random initial conditions $X(0)=Y_{0}$ and $\dot{X}(0)=Y_{1}$ is given by

$$
X(t)=Y_{0} X_{1}(t)+Y_{1} X_{2}(t), \quad t \in(-\infty,+\infty),
$$

where $X_{1}(t)$ and $X_{2}(t)$ are defined in $(27)$.

Summarizing the following result has been established:

Theorem 13 The random differential equation (1) with initial conditions $X(0)=Y_{0}$ and $\dot{X}(0)=Y_{1}$, where $A$ is a r.v. satisfying condition (18), admits as random power series solution of the form (33) where $X_{1}(t)$ and $X_{2}(t)$ are given by (27). Moreover the solution is m.s. convergent for each $t \in(-\infty,+\infty)$.

Remark 14 The constructive method we have developed previously justifies itself the existence and uniqueness of the analytical m.s. solution of the random differential equation (1) with initial conditions $X(0)=Y_{0}$ and $\dot{X}(0)=Y_{1}$. In fact, from the initial conditions $X_{0}=Y_{0}$ and $X_{1}=Y_{1}$, the recurrence (23) permits to compute, in a unique way, the rest of the coefficients $X_{n}, n \geq 2$ that define the s.p. solution (26)-(27). In addition, we have proven that this random power series is m.s. convergent for every $t$. On the one hand, notice that in this case the approach above permits to guarantee the existence and uniqueness of the analytical m.s. solution of problem (1) with initial conditions $X(0)=Y_{0}$ and $\dot{X}(0)=Y_{1}$ in an easier way than applying the natural generalization to the random framework of the classical Picard-type theorem for general differential equations based upon convergence of successive approximations established in theorem 5.1 .1 of [5, p.118] since its application requires to check a m.s. Lipschitz condition which in practice is too restrictive (see [5, p.119] for comments on this issue). On the other hand, we point out that although theorem 8.1.1. of [5, p.219] provides an analogous result to theorem 5.1 .1 but under weaker conditions (because it is just suited to linear random differential equations) it only allows us to guarantees m.s. convergence in a neighborhood about $t=0$ whose radius will likely be very small in order to assure the required convergence of the numerical series given in condition (b). In connection with our development, notice that this condition (b) essentially relies on the growth rates of the statistical moments associated with the input r.v. A such as we have assumed in condition (18) (see [5, p.219] for additional comments on this issue).

From (31), one deduces that previous exposition holds true by assuming the following growth condition about the absolute moments of r.v. $A$

$$
\exists k_{1}: \mathrm{E}\left[|A|^{2^{k+2}}\right] \leq(4 k)^{2^{k+2}}, \quad \forall k \geq k_{1},
$$

which is less restrictive that (18). However, from a practical viewpoint previous 
condition is not easy to check because of the lack of explicit expressions for the absolute moments with respect to the origin of relevant r.v.'s such as Binomial, Poisson, etc. In order to overcome this difficulty, from now on, we will deal with r.v.'s $A$ having finite domain, i.e., such that $a_{1} \leq A(\omega) \leq a_{2}$, for each $\omega \in \Omega$. Note that this class of r.v.'s satisfy condition (18). Indeed, let us assume that $A$ is a continuous r.v. with density function $f_{A}(a)$, then taking $H=\max \left(\left|a_{1}\right|,\left|a_{2}\right|\right)$, one gets

$$
\mathrm{E}\left[|A|^{n}\right]=\int_{a_{1}}^{a_{2}}|a|^{n} f_{A}(a) d a \leq H^{n} .
$$

Note that, by substituting the integral for a sum, previous conclusion remains true if $A$ is a discrete r.v. We emphasize that important r.v.'s such as Binomial, Hypergeometric, Uniform or Beta have finite domain. Otherwise, we can use the truncation method (see [12]) for dealing with unbounded r.v.'s such as Poisson, Gaussian or Exponential (see Example 19 below).

\section{Introducing the random Hermite polynomials}

Next we address to motivate the introduction of random Hermite polynomials as well as some aspects related to them that arise in a natural way when we extend this deterministic concept to the stochastic framework. First, note that taking into account (23)-(27), one deduces that if there exists $n \geq 0$ such that $\mathrm{P}[A=2 n]=1$, that is, $A$ is a (degenerate) discrete r.v. whose total probability mass is concentrated at the point $2 n$, then the random differential equation (1) has a random polynomial solution. Since every even integer number takes the form $4 k$ or $4 k+2, k \geq 0$, if there exists $k \geq 0$ such that $\mathrm{P}[A=4 k]=1$ (or $\mathrm{P}[A=4 k+2]=1)$, then $X_{1}(t)$ (or $\left.X_{2}(t)\right)$ given by $(27)$ generates a (random) polynomial solution of degree $2 k$ (or $2 k+1$ ). These random (degenerate) solutions can be interpreted as corresponding Hermite polynomials that one presents in the deterministic framework. However in the random scenario there are richer situations that deserve to be considered.

Indeed, in the case that $A$ is a continuous r.v. since $\mathrm{P}[A=2 n]=0$ for every integer $n \geq 0$, then with probability 1 , one can conclude that there are not random polynomial solutions of differential equation (1). Whereas if $A$ is a discrete r.v. that only takes different even values (not concentrated in just one even value), then there will exist with probability 1 , random polynomial solutions (see later, Example 20). This case generalizes the concept of Hermite polynomial solution from the deterministic framework (moreover, note that this situation contains the previous case where $A$ was a degenerated r.v. concentrated just at a point).

For the case that $A$ is a discrete r.v. whose values lie in a set containing some 
even numbers, random differential equation (1) does not have any random polynomial solution, but it admits some sample representations which are (deterministic) polynomials. Then, considering the stochastic process solution as a family of trajectories, we can assign the probability $p_{p o l}$ that the random power series given by (33) and (27) has random polynomial sample solutions. Note that this series becomes a random polynomial if, and only if, $p_{p o l}=1$. Thus if $0 \leq p_{\text {pol }}<1$, then $X(t)$ given by (33) and (27) produces sample solutions, with probability $p_{\text {pol }}$, of random differential equation (1) with initial conditions $Y_{0}(\omega), Y_{1}(\omega)$, but not a random solution.

As an illustrative example, let us assume that $A$ is Binomial r.v. of parameters $n$ and $p, A \sim \operatorname{Bi}(n ; p)$. Table 1 shows the probability $p_{p o l}$ for different values of the parameters $n$ and $p$. As in general, $\operatorname{Bi}(n ; p)=\operatorname{Bi}(n ; 1-p)$, we only consider values $0<p \leq 0.5$. One observes that for $p$ fixed, these values decrease up to 0.5 as $n$ increases. The considerations above motivate the following result:

\begin{tabular}{|c|c|c|c|c|c|}
\hline$n$ & $p_{\text {pol }}, p=0.1$ & $p_{\text {pol }}, p=0.2$ & $p_{\text {pol }}, p=0.3$ & $p_{\text {pol }}, p=0.4$ & $p_{\text {pol }}, p=0.5$ \\
\hline 2 & 0.82 & 0.68 & 0.58 & 0.52 & 0.5 \\
\hline 4 & 0.7048 & 0.5648 & 0.5128 & 0.5008 & 0.5 \\
\hline 6 & 0.631072 & 0.523328 & 0.502048 & 0.500032 & 0.5 \\
\hline 8 & 0.583886 & 0.508398 & 0.500328 & 0.500001 & 0.5 \\
\hline 10 & 0.553687 & 0.503023 & 0.500052 & 0.5 & 0.5 \\
\hline 100 & 0.5 & 0.5 & 0.5 & 0.5 & 0.5 \\
\hline
\end{tabular}

Table 1

Probabilities of generating random polynomial (sample) solutions when $A$ is a Binomial r.v. of parameters $n$ and $p$

Corollary 15 Let us consider the random differential equation (1) with initial conditions $X(0)=Y_{0}$ and $\dot{X}(0)=Y_{1}$, where the (discrete) r.v. A takes only a finite number of even integer values, that is, $\mathrm{P}\left[A=2 m_{j}\right]=p_{j}>0,1 \leq j \leq n$ with $\sum_{j=1}^{n} p_{j}=1$. Then this i.v.p. has a random polynomial solution $H_{m_{n}}(t)$ of the degree $m_{n}$.

Definition 16 Let $m_{n}$ be a positive integer. The $m_{n}$-th random Hermite polynomial or the random Hermite polynomial of degree $m_{n}$, is the random m.s. solution of problem (1) with initial conditions $X(0)=Y_{0}$ and $\dot{X}(0)=Y_{1}$, being $A$ the discrete r.v. taking the finite number of even integer values $2 m_{j}$, with probabilities $\mathrm{P}\left[A=2 m_{j}\right]=p_{j}>0,1 \leq j \leq n$ with $\sum_{j=1}^{n} p_{j}=1$.

With respect to Definition 16 and keeping this notation, it is important to point out that under conditions of Corollary 15, a random Hermite polynomial solution can be interpreted as a collection of deterministic Hermite polynomials, which, for each $j: 1 \leq j \leq n$, have a probability $p_{j}$ of sampling. 
The degree $m_{n}$ of the random Hermite polynomial $H_{m_{n}}(t)$ is the greatest of all degrees corresponding to each (deterministic) Hermite polynomials, but it is not necessary that its sample associated probability $p_{n}$ be also greater than $p_{j}$ for all $j: 1 \leq j \leq n$, (see Example 20).

Remark 17 Note that the situation where $A$ is the discrete r.v. taking all the even integer values with $P[A=2 j]=2^{-(j+1)}, j=0,1,2, \ldots$, then for each $j$ one obtains a sample polynomial solution, but since $j$ lies in the positive integer numbers, the degree of the random polynomial solution cannot be defined according with Definition 16.

\section{Statistical functions of the mean square random power series solution}

This section deals with the computation of the main statistical functions of the m.s. solution of (1) given by (26)-(27) such that the average and variance in terms of the data $\mathrm{E}\left[Y_{0}\right], \mathrm{E}\left[Y_{1}\right], \mathrm{E}\left[Y_{0} Y_{1}\right], \mathrm{E}\left[\left(Y_{0}\right)^{2}\right], \mathrm{E}\left[\left(Y_{1}\right)^{2}\right]$ as well as certain moments related to algebraic transformations of the random coefficient $A$ that will be specified later.

First at all, let us take the expectation operator in the random differential equation (1). Then by applying property (8) one gets

$$
\frac{d^{2}}{d t^{2}}\left(\mu_{X}(t)\right)-2 t \frac{d}{d t}\left(\mu_{X}(t)\right)+\mathrm{E}[A X(t)]=0 .
$$

Note that, (35) is not a suitable equation for computing $\mu_{X}(t)$ because the term $\mathrm{E}[A X(t)]$ cannot be factorized as $A \mu_{X}(t)$, in general. Nevertheless some methods, like the so-called dishonest method (see, [19], [20, p.148]), accept the above factorization as an alternative to handle the problem of computing the mean of the solution process. Since we shall see right away through examples, our approach avoid the above approximations and it allows us to provide reliable values for the mean and the variance into quite general situations.

In practice, as it occurs in the deterministic framework, it will be unfeasible the computation of the mean through the infinite series given by (26)-(27). Then, we will consider the truncation of order $N$

$$
\begin{aligned}
X_{N}(t) & =X_{0}\left(1+\sum_{k=0}^{N} \frac{t^{2 k+2}}{(2 k+2) !} \prod_{j=0}^{k}(4 j-A)\right) \\
& +X_{1}\left(t+\sum_{k=0}^{N} \frac{t^{2 k+3}}{(2 k+3) !} \prod_{j=0}^{k}(4 j+2-A)\right) .
\end{aligned}
$$


Henceforth, we will assume that r.v. $A$ is independent of initial conditions $X(0)=Y_{0}$ and $\dot{X}(0)=Y_{1}$ (note that from an applied point of view, this hypothesis is realistic), then taking the expectation operator in (36) one gets

$$
\begin{aligned}
& \mu_{X_{N}}(t)=\mathrm{E}\left[Y_{0}\right]\left(1+\sum_{k=0}^{N} \frac{t^{2 k+2}}{(2 k+2) !} \mathrm{E}\left[\prod_{j=0}^{k}(4 j-A)\right]\right) \\
&+\mathrm{E}\left[Y_{1}\right]\left(t+\sum_{k=0}^{N} \frac{t^{2 k+3}}{(2 k+3) !} \mathrm{E}\left[\prod_{j=0}^{k}(4 j+2-A)\right]\right) . \\
& \mathrm{E}\left[\prod_{j=0}^{k}(4 j-A)\right]=\left\{\begin{array}{l}
\sum_{a: p_{A}(a)>0} \prod_{j=0}^{k}(4 j-a) p_{A}(a), \\
\int_{-\infty}^{\infty} \prod_{j=0}^{k}(4 j-a) f_{A}(a) d a
\end{array}\right.
\end{aligned}
$$

and

$$
\mathrm{E}\left[\prod_{j=0}^{k}(4 j+2-A)\right]=\left\{\begin{array}{c}
\sum_{a: p_{A}(a)>0} \prod_{j=0}^{k}(4 j+2-a) p_{A}(a), \\
\int_{-\infty}^{\infty} \prod_{j=0}^{k}(4 j+2-a) f_{A}(a) d a .
\end{array}\right.
$$

Now taking into account the expression (6) for computing the variance of the truncated solution process, now we only require to calculate $\mathrm{E}\left[\left(X_{N}(t)\right)^{2}\right]$. In order to save executing time in the computer, it is convenient considering the following relationship

$$
\mathrm{E}\left[\left(X_{N}(t)\right)^{2}\right]=\sum_{n=0}^{N} \mathrm{E}\left[\left(X_{n}\right)^{2}\right] t^{2 n}+2 \sum_{n=1}^{N} \sum_{m=0}^{n-1} \mathrm{E}\left[X_{n} X_{m}\right] t^{n+m} .
$$

With the usual convection $\prod_{i=u}^{v} f(i)=1$ if $v<u$, the terms involved in the two previous sums can be computed as follows:

$$
\mathrm{E}\left[X_{n} X_{m}\right]=\frac{1}{n ! m !}\left\{\begin{array}{l}
\mathrm{E}\left[\left(Y_{0}\right)^{2}\right] \mathrm{E}\left[P_{1}\left(\frac{n-2}{2}\right) P_{1}\left(\frac{m-2}{2}\right)\right] \text { if } \begin{array}{l}
n=0,2,4, \ldots, \\
m=0,2,4, \ldots,
\end{array} \\
\mathrm{E}\left[Y_{0} Y_{1}\right] \mathrm{E}\left[P_{1}\left(\frac{n-2}{2}\right) P_{2}\left(\frac{m-3}{2}\right)\right] \text { if } \begin{array}{l}
n=0,2,4, \ldots, \\
m=1,3,5, \ldots,
\end{array} \\
\mathrm{E}\left[Y_{0} Y_{1}\right] \mathrm{E}\left[P_{2}\left(\frac{m-3}{2}\right) P_{1}\left(\frac{n-2}{2}\right)\right] \text { if } \begin{array}{l}
n=1,3,5, \ldots, \\
m=0,2,4, \ldots,
\end{array} \\
\left.\left.\begin{array}{l}
m=1,3,5, \ldots, \\
n
\end{array}\right)^{2}\right] \mathrm{E}\left[P_{2}\left(\frac{n-3}{2}\right) P_{2}\left(\frac{m-3}{2}\right)\right] \text { if } \begin{array}{l}
n=1,3,5, \ldots, \\
m=1
\end{array}
\end{array}\right.
$$


where we have denoted

$$
P_{1}(k)=\prod_{j=0}^{k}(4 j-A), \quad P_{2}(k)=\prod_{j=0}^{k}(4 j+2-A),
$$

and the expectations appearing in (38) can be computed as follows

$$
\begin{gathered}
\mathrm{E}\left[P_{1}(k) P_{1}(l)\right]=\left\{\begin{array}{c}
\sum_{a: p_{A}(a)>0} \prod_{j=0}^{k}(4 j-a) \prod_{j=0}^{l}(4 j-a) p_{A}(a), \\
\int_{-\infty}^{+\infty} \prod_{j=0}^{k}(4 j-a) \prod_{j=0}^{l}(4 j-a) f_{A}(a) d a,
\end{array}\right. \\
\mathrm{E}\left[P_{1}(k) P_{2}(l)\right]=\left\{\begin{array}{l}
\sum_{a: p_{A}(a)>0} \prod_{j=0}^{k}(4 j-a) \prod_{j=0}^{l}(4 j+2-a) p_{A}(a), \\
\int_{-\infty}^{+\infty} \prod_{j=0}^{k}(4 j-a) \prod_{j=0}^{l}(4 j+2-a) f_{A}(a) d a,
\end{array}\right. \\
\mathrm{E}\left[P_{2}(k) P_{1}(l)\right]=\left\{\begin{array}{c}
\sum_{a: p_{A}(a)>0} \prod_{j=0}^{k}(4 j+2-a) \prod_{j=0}^{l}(4 j-a) p_{A}(a), \\
\int_{-\infty}^{+\infty} \prod_{j=0}^{k}(4 j+2-a) \prod_{j=0}^{l}(4 j-a) f_{A}(a) d a,
\end{array}\right. \\
\mathrm{E}\left[P_{2}(k) P_{2}(l)\right]=\left\{\begin{array}{c}
\sum_{a: p_{A}(a)>0} \prod_{j=0}^{k}(4 j+2-a) \prod_{j=0}^{l}(4 j+2-a) p_{A}(a), \\
\int_{-\infty}^{+\infty} \prod_{j=0}^{k}(4 j+2-a) \prod_{j=0}^{l}(4 j+2-a) f_{A}(a) d a .
\end{array}\right.
\end{gathered}
$$

Taking into account property (7) as well as the m.s. convergence of random series given by (33) and (27), the convergence of the mean and the variance of the truncated solution (36) to the corresponding exact values are warranted under the hypotheses of Theorem 13.

\section{$7 \quad$ Examples}

In this section we provide several illustrative examples. The results obtained to approximate the mean and the variance by means of the series method presented in this paper are compared with respect to the corresponding ones provided by the dishonest and Monte Carlo approaches.

Example 18 Let us consider a random differential equation of the form (1) where $A$ is a Beta r.v. with parameters $\alpha=2$ and $\beta=3$, i.e., $A \sim B e(\alpha=$ $2 ; \beta=3)$ and the initial conditions $Y_{0}$ and $Y_{1}$ are (positive) correlated r.v.'s 
such that $\mathrm{E}\left[Y_{0} Y_{1}\right]=0.3$ and, $\mathrm{E}\left[Y_{0}\right]=1, \mathrm{E}\left[\left(Y_{0}\right)^{2}\right]=2, \mathrm{E}\left[Y_{1}\right]=2, \mathrm{E}\left[\left(Y_{1}\right)^{2}\right]=$ 5. Note that r.v. A satisfies conditions of Theorem 13 since it takes values on a bounded interval. Then the m.s. solution of model (1) with initial conditions $Y_{0}$ and $Y_{1}$ is given by (33) and (27). Table 2 shows the expectation of the truncated solution s.p. for different values of the truncation order $N$ (denoted by $\mu_{X_{N}}(t)$ ) at different values of the time parameter $t$ as well as the corresponding values obtained by the dishonest $\left(\mu_{X}^{d}(t)\right)$ and Monte Carlo methods $\left(\widetilde{\mu}_{X}^{m}(t)\right)$ by using $m$ simulations. One observes that for values of $t$ near of the origin (where

\begin{tabular}{|c|c|c|c|c|c|c|}
\hline$t$ & $\mu_{X_{5}}(t)$ & $\mu_{X_{10}}(t)$ & $\mu_{X_{20}}(t)$ & $\mu_{X}^{d}(t)$ & $\begin{array}{c}\widetilde{\mu}_{X}^{m}(t) \\
m=50000\end{array}$ & $\begin{array}{c}\widetilde{\mu}_{X}^{m}(t) \\
m=100000\end{array}$ \\
\hline 0.00 & 1.00000 & 1.00000 & 1.00000 & 1.00000 & 0.99826 & 0.99995 \\
\hline 0.25 & 1.49575 & 1.49575 & 1.49575 & 1.49574 & 1.49502 & 1.49611 \\
\hline 0.50 & 2.01775 & 2.01775 & 2.01775 & 2.01761 & 2.01814 & 2.01855 \\
\hline 0.75 & 2.63184 & 2.63184 & 2.63184 & 2.63099 & 2.63362 & 2.63315 \\
\hline 1.00 & 3.44890 & 3.44891 & 3.44891 & 3.44545 & 3.45263 & 3.45088 \\
\hline 1.25 & 4.69234 & 4.69267 & 4.69267 & 4.68111 & 4.69942 & 4.69553 \\
\hline 1.50 & 6.86741 & 6.87373 & 6.87373 & 6.83843 & 6.88590 & 6.87801 \\
\hline 1.75 & 11.2081 & 11.2854 & 11.2855 & 11.1806 & 11.30880 & 11.29240 \\
\hline 2.00 & 20.8513 & 21.5482 & 21.5514 & 21.2341 & 21.60060 & 21.56350 \\
\hline
\end{tabular}

Table 2

Comparison of the mean for different methods in Example 18

the initial conditions are established and the series solution s.p. is centered), the approximations obtained by the method proposed in this paper coincide for different truncation orders of the series solution. In fact, the approximations are accurate for $N=5$ about $t=0$. These values differ from the corresponding ones obtained by the dishonest method. Regarding approximations obtained by means of the Monte Carlo method it is worthwhile pointing out that they improve as the number $m$ of simulations increases, and in general, they provide better approximations than those obtained by the dishonest method. Table 3 compares the values of variance for the truncation method with respect to the Monte Carlo method. In order to show that accurate approximations of the variance require greater values of $N$, in Table 3 we have considered values of $N$ that differ from those we have taken in Table 2. Finally, note that as $A$ is a continuous r.v., in this case the initial value problem does not have random (Hermite) polynomial solutions.

Example 19 In this example we take advantage of the so-called truncation method (see [12]) to deal with a r.v. A that neither satisfy condition (18) nor condition (34). Let us consider model (1) where $A$ is a Gaussian r.v., 


\begin{tabular}{|c|c|c|c|c|c|}
\hline$t$ & $\operatorname{Var}\left[X_{10}(t)\right]$ & $\operatorname{Var}\left[X_{20}(t)\right]$ & $\operatorname{Var}\left[X_{50}(t)\right]$ & $\begin{array}{c}\widetilde{\operatorname{Var}}_{X}(t) \\
m=50000\end{array}$ & $\begin{array}{c}\widetilde{\operatorname{Var}}_{X}^{m}(t) \\
m=100000\end{array}$ \\
\hline 0.00 & 1.00000 & 1.00000 & 1.00000 & 1.002 & 0.9978086 \\
\hline 0.25 & 1.03943 & 1.03943 & 1.03943 & 1.04321 & 1.03741 \\
\hline 0.50 & 1.18425 & 1.18425 & 1.18425 & 1.18964 & 1.18298 \\
\hline 0.75 & 1.54404 & 1.54432 & 1.54432 & 1.55086 & 1.54517 \\
\hline 1.00 & 2.44517 & 2.45458 & 2.45458 & 2.4609 & 2.461183 \\
\hline 1.25 & 4.84512 & 5.01194 & 5.01197 & 5.01346 & 5.035022 \\
\hline 1.50 & 11.2801 & 13.3658 & 13.3675 & 13.3465 & 13.4437 \\
\hline 1.75 & 24.4765 & 46.25617 & 46.3373 & 46.2146 & 46.61740 \\
\hline 2.00 & 105.1987 & 206.11745 & 209.017 & 208.359 & 210.273 \\
\hline
\end{tabular}

Table 3

Comparison of the variance for different methods in Example 18

$A \sim \mathrm{N}(\mu=5 ; \sigma=1)$, and the initial conditions $Y_{0}$ and $Y_{1}$ are uncorrelated r.v.'s such that $\mathrm{E}\left[Y_{0}\right]=1, \mathrm{E}\left[\left(Y_{0}\right)^{2}\right]=2, \mathrm{E}\left[Y_{1}\right]=2, \mathrm{E}\left[\left(Y_{1}\right)^{2}\right]=5$. Note that r.v. A has unbounded domain. In order to overcome this difficulty, we will consider the truncation of this r.v. on the interval $[\mu-3 \sigma, \mu+3 \sigma]=[2,8]$ that will contain all the values of $A$ with probability 0.997 . The probability density function associated to the new censured r.v., say, $B$ is

$$
f_{B}(b)=\frac{\exp \left(-\frac{1}{2}(b-5)^{2}\right)}{\int_{2}^{8} \exp \left(-\frac{1}{2}(x-5)^{2}\right) d x}, \quad 2 \leq b \leq 8 .
$$

In this way, B satisfies hypotheses of Theorem 13 since it takes values on a bounded interval. Table 4 shows approximations of the expectation of the solution s.p. computed by the truncation series, dishonest and Monte Carlo methods. For the truncation method, Table 4 only shows results for $N=5,10$ because for greater values the obtained results do not change. Table 5 shows approximations for the variance obtained by means of the truncation method as well as Monte Carlo method. Analogous comments we made in Example 18 can be done again.

Example 20 Let us consider model (1) where $A$ is a discrete r.v. whose values and associated probabilities are specified in the two first columns of Table 6. In this case, the random differential equation (1) with initial conditions $X(0)=$ $Y_{0}$ and $\dot{X}(0)=Y_{1}$ has random (Hermite) polynomial solutions of degree 9 . For instance, with probability $1 / 8$ one will present as (sample) solution the polynomial $1-2 t^{2}$ generated from the term $X_{1}(t)$ (given by (27)) that defines the general solution (26). Apart from a constant, this polynomial coincides 


\begin{tabular}{|c|c|c|c|c|c|}
\hline$t$ & $\mu_{X_{5}}(t)$ & $\mu_{X_{10}}(t)$ & $\mu_{X}^{d}(t)$ & $\begin{array}{c}\widetilde{\mu}_{X}^{m}(t) \\
m=50000\end{array}$ & $\begin{array}{c}\widetilde{\mu}_{X}^{m}(t) \\
m=100000\end{array}$ \\
\hline 0.00 & 1.00000 & 1.00000 & 1.00000 & 0.996945 & 0.99917 \\
\hline 0.25 & 1.32907 & 1.32907 & 1.32889 & 1.3267 & 1.3286 \\
\hline 0.50 & 1.26473 & 1.26473 & 1.26175 & 1.26405 & 1.26507 \\
\hline 0.75 & 0.7451 & 0.745101 & 0.729061 & 0.747267 & 0.746903 \\
\hline 1.00 & -0.271569 & -0.271568 & -0.324665 & -0.265094 & -0.267331 \\
\hline 1.25 & -1.80638 & -1.80635 & -1.93991 & -1.7937 & -1.79838 \\
\hline 1.50 & -3.85904 & -3.85876 & -4.13681 & -3.83768 & -3.84559 \\
\hline 1.75 & -6.41039 & -6.4087 & -6.90081 & -6.37705 & -6.38944 \\
\hline 2.00 & -9.43986 & -9.43401 & -10.1448 & -9.39179 & -9.4106 \\
\hline
\end{tabular}

Table 4

Comparison of the mean for different methods in Example 19

\begin{tabular}{|c|c|c|c|c|c|}
\hline$t$ & $\operatorname{Var}\left[X_{10}(t)\right]$ & $\operatorname{Var}\left[X_{20}(t)\right]$ & $\operatorname{Var}\left[X_{50}(t)\right]$ & $\begin{array}{c}\widetilde{\operatorname{Var}}_{X}^{m}(t) \\
m=50000\end{array}$ & $\begin{array}{c}\widetilde{\operatorname{Var}}_{X}(t) \\
m=100000\end{array}$ \\
\hline 0.00 & 1.00000 & 1.00000 & 1.00000 & 0.99837 & 0.998648 \\
\hline 0.25 & 0.774331 & 0.774331 & 0.774331 & 0.77285 & 0.774595 \\
\hline 0.50 & 0.37752 & 0.37752 & 0.37752 & 0.379991 & 0.380405 \\
\hline 0.75 & 0.54182 & 0.541811 & 0.541811 & 0.550826 & 0.546854 \\
\hline 1.00 & 2.10416 & 2.10396 & 2.10396 & 2.11649 & 2.10742 \\
\hline 1.25 & 5.48791 & 5.48669 & 5.48669 & 5.49385 & 5.48397 \\
\hline 1.50 & 10.4335 & 10.4463 & 10.4463 & 10.454 & 10.4573 \\
\hline 1.75 & 17.6092 & 18.0106 & 18.0117 & 18.1235 & 18.1765 \\
\hline 2.00 & 38.0585 & 43.7945 & 43.8404 & 44.5363 & 44.7127 \\
\hline
\end{tabular}

Table 5

Comparison of the variance for different methods in example 19

with the deterministic Hermite polynomial, given by $4 t^{2}-2$. In fact, it is enough taking as initial condition $Y_{0}$ a r.v. concentrated in the point -2, i.e., $P\left[Y_{0}=-2\right]=1$ for obtaining the Hermite (sample) polynomial of degree 2, $4 t^{2}-2$. In the same way, with probability $1 / 4$ we will obtain as solution the polynomial $t-2 / 3 t^{3}$ which is generated from the term $X_{2}(t)$ given by (27). For an initial condition $Y_{1}$ such that $P\left[Y_{1}=-12\right]=1$ one obtain just the deterministic Hermite polynomial of degree 3, $8 t^{3}-12 t$. Following an analogous procedure, we fill the table 6 . We stress the degree of the random polynomial 
solution is 9 and, the sample polynomial solution associated to this degree is $1 / 6$, whereas the sample polynomial solution of degree 5 has the greater probability of occurrence, $1 / 3$.

\begin{tabular}{|c|c|c|c|}
\hline$A$ & $P[A]$ & initial condition & Hermite sample polynomial \\
\hline 4 & $1 / 8$ & $Y_{0}=-2$ & $-2+4 t^{2}$ \\
\hline 6 & $1 / 4$ & $Y_{1}=-12$ & $-12 t+8 t^{3}$ \\
\hline 10 & $1 / 3$ & $Y_{1}=120$ & $120 t-160 t^{3}+32 t^{5}$ \\
\hline 12 & $1 / 8$ & $Y_{0}=-120$ & $-120+720 t^{2}-480 t^{4}+64 t^{6}$ \\
\hline 18 & $1 / 6$ & $Y_{1}=30240$ & $30240 t-80640 t^{3}+48384 t^{5}-9216 t^{7}+512 t^{9}$ \\
\hline
\end{tabular}

Table 6

Hermite sample polynomials and its associated probabilities in Example 20

\section{Conclusions}

In this paper, we have constructed a power series solution of the Hermite random differential equation (1) by assuming that coefficient $A$ is a random variable satisfying condition (18) related to its statistical moments. In order to justify that this power series is mean square convergent over the whole real line, the inequality (17) as well as some results linking the mean square and mean fourth calculus have been previously established. We have also provided sufficient conditions for obtaining random polynomial solutions of (1) which allows to introduce the random Hermite polynomials as an extension of their deterministic counterpart. On the other hand, by assuming independence between random initial conditions and input random variable $A$, explicit analytic-numerical approximations of the main statistical functions associated to the stochastic process solution of (1) have been provided. In the last section, we have shown several illustrative examples where the approximations of the mean and the variance of the solution of (1) with respect to dishonest and Monte Carlo approaches are compared. These examples show that the approach here proposed is more accurate.

\section{Acknowledgement}

This work has been partially supported by the Spanish M.C.Y.T. and FEDER grants MTM2009-08587, DPI2010-20891-C02-01 as well as the Universitat Politècnica de València grant PAID-06-09 (ref. 2588). 


\section{References}

[1] J.L. Strand, Random ordinary differential equations, J. Diff. Equat. 7 (1973) $538-553$.

[2] L. Arnold, Stochastic Differential Equations Theory and Applications, John Wiley, New York 1974.

[3] B. Øksendal, Stochastic Differential Equations. An Introduction with Applications 5th Ed., Springer-Verlag, Berlin Heildelberg 1998.

[4] J.L. Strand, Stochastic Ordinary Differential Equations, Ph.D. Thesis, Univ. of California, Berkeley, California 1968.

[5] T.T. Soong, Random Differential Equations in Science and Engineering, Academic Press, New York 1973.

[6] J.C. Cortés, L. Jódar, L. Villafuerte, Random linear-quadratic mathematical models: computing explicit solutions and applications, Math. Comput. Simulat. 79(7) (2009) 2076-2090.

[7] M.A. El-Tawil, The approximate solutions of some stochastic differential equations using transformations, Appl. Math. Comput., 164(1) (2005) 167-178.

[8] L. Villafuerte, C.A. Braumann, J.C. Cortés, L. Jódar, Random differential operational calculus: Theory and applications, Comput. Math. Appl. 59 (2010) $115-121$.

[9] J.C. Cortés, L. Jódar, L. Villafuerte, Numerical solution of random differential initial value problems: Multistep methods, Math. Meth. Appl. Sci. 34(1) (2011) $63-75$.

[10] M. El-Tawil, W. El-Tahan, A. Hussein A proposed technique of SFEM on solving ordinary random differential equation, Appl. Math. Comput. 161 (2005) $35-47$.

[11] G. Calbo, J.C. Cortés, L. Jódar, Random analytic solution of coupled differential models with uncertain initial condition and source term, Comput. Math. Appl. 56 (2008) 785-798.

[12] M. Loève, Probability Theory, Van Nostrand, Princeton, New Jersey 1963.

[13] E. Wong, B. Hajek, Stochastic Processes in Engineering System, Springer Verlag, New York 1985.

[14] S. Elaydi, An Introduction to Difference Equations, Third Ed., Springer, New York 2005.

[15] I.S. Gradshteyn, I.M. Ryzhik, Table of Integrals, Series and Products, Academic Press, England 1994.

[16] N. N. Lebedev, Special Functions and their Applications, Dover, New York 1972. 
[17] G.R. Grimmett, D.R. Stirzaker, Probability and Random Processes, Claredon Press, Oxford 2000.

[18] J.C. Cortés, P. Sevilla-Peris, L. Jódar, Analytic-numerical approximating processes of diffusion equation with data uncertainty, Comput. Math. Appl. 49 (2005) 1255-1266.

[19] J.B. Keller, Stochastic equations and wave propagation in random media, Procc. Symp. Appl. Math. Am. Math. Soc. Providence Rhode Island 196316 (1963) $145-170$.

[20] D. Henderson, P. Plaschko, Stochastic Differential Equations in Science and Engineering, World Scientific, Singapore 2006. 